\title{
CONDIÇÕES DE METAMORFISMO NO COMPLEXO GRANULÍTICO SANTA MARIA CHICO, RS
}

\author{
LEO A. HARTMANN*
}

\begin{abstract}
METAMORPHIC CONDITIONS IN THE SANTA MARIA CHICO GRANULITE COMPLEX, RS. Granulite-facies rocks froin Western Rio Grande do Sul in southernmosl Brazil are bimodal acid and basic in composition, wilh sinaller ainounls of interinediate and ultrabasic compositions; supracrustal relics include quartzites, marbles and sillimanite gneisses. The first granulite-facies event is only registered as inlensely exsolved megacrysts of pyroxenes, plagioclase and other minerais. A third metamorphic episode was caused by the intrusion of abundant granites, general i ng actinolite, hornblende, clorite and tale from the mafic minerais. Microprobe analyses show the a.i.phiboles as pargasite in the $\mathrm{M}_{2}$ high-grade event and the plagioclase as andesine, labradorite, bylownile and anorthite. This event was dated at $2.1 \mathrm{Ga}$ by $\mathrm{Sm} / \mathrm{Nd}$ isofopes and the intrusion of granites occurred at $550 \mathrm{Ma}$. Temperatura and pressure of the M2 metamorphic event were estimated, using several thermometers and barometers. The temperature is estimated at $800-850^{\circ} \mathrm{C}$ and the pressure at $9.4 \mathrm{kbar}$.
\end{abstract}

Keywords: Granulite, geolhennobaroinetry, mineral chemistry.

\begin{abstract}
RESUMO As litologias de fácies granulito ocorrentes no oeste do Rio Grande do Sul são bimodais básicas e ácidas em composição, com volumes menores de roclias intermediárias e ultrabásicas; remanescentes supracrustais incluem quartzitos, mármores e sillimanita gnaisses. O primeiro evento de fácies granulito está registrado somente por megacristais de piroxênio, plagioclásio e outros minerais, sempre com fortes feições de exsolução.Um terceiro episódio metamórfico foi causado pela intrusão de abundantes grainitos, gerando aclinolita, hornblenda, clorita e talco a partir dos minerais máfícos. Análises por microssonda eletrônica mostram que os anfibólios são pargasitas no evento $\mathrm{M}_{2}$ de alto grau, e que os plagioclásios são andesina, labradorita, bytownita e anorlita. Este evento foi datado em 2, 1 Ga por Sn./Nd em minerais separados, ao passo que a intrusão dos grani.os ocorreu há 550 Ma. A temperatura e a pressão do evento $\mathrm{M}_{2}$ foram estimadas utilizando-se vários termómetros e barómetros. A temperatura é estimada em $800-850^{\circ} \mathrm{C}$ e a pressão em 9,4 kbar.
\end{abstract}

Palavras-chaves: Granulito, geotermobarometria, química mineral.

INTRODUÇÃO O Complexo Granulítico Santa Maria Chico, inicialmente caracterizado por Nardi \& Hartmann (1979), ocorre no extremo oeste da área exposta de rochas pré-cambrianas no Rio Grande do Sul. O complexo tem sido mapeado por formandos dos cursos de geologia da Universidade Federal do Rio Grande do Sul e da Universidade do Vale do Rio dos Sinos, que caracterizaram as relações geológicas principais. Naumann et al (1984) assinalaram as relações geológicas na parte norte do complexo. Revisões regionais foram feitas por Jost \& Hartmann (1984) e Santos et al (1984). Estudos geocronológicos com utilização de isótopos de $\mathrm{Rb} / \mathrm{Sr}$ e K/Ar foram realizados por Soliani (1986). Hartmann (1988) avalia dados de elementos terras raras e apresenta uma seleção de dados de geotermobarometria do complexo. Datação de minerais separados com isótopos de $\mathrm{Sm} / \mathrm{Nd}$ foi publicada por Hartmann (1987), fornecendo 2,1 Ga para a idade do metamorfismo granulítico mais bem registrado. Figueiredo \& Hartmann (1989) compararam o comportamento dos elementos terras raras do complexo com outros terrenos granulíticos do Brasil.

No artigo de Hartmann (1988), são avaliados alguns aspectos geológicos e petrográficos do complexo. Neste trabalho, são apresentados e discutidos dados químicos de minerais e cálculos geotermobarométricos decorrentes. Os dados químicos foram obtidos em microssonda eletrônica Cambridge Instruments da Open University, Inglaterra, em condições de $20 \mathrm{kV}$, feixe desfocado. Os cálculos de pressão e temperatura foram realizados com a utilização de computador dessa universidade inglesa.

ASPECTOS MINERALÓGICOS A mineralogia presente no complexo é de origem metamórfica, não tendo sido identificados minerais ou texturas ígneas remanescentes. $\mathrm{O}$ evento metamórfico $\mathrm{Mi}$, de fácies granulito, está registrado pela presença de porfiroclastos de piroxênios e plagioclásio. Esses minerais não foram analisados, devido à presença de lamelas de exsolução. A possibilidade de que se trata de remanescentes de mineralogia ígnea não pode no entanto, ser descartada.

$\mathrm{O}$ evento $\mathrm{M}_{2}$, de metamorfismo regional de fácies granulito, está bem desenvolvido, formando a mineralogia dominante, com ortopiroxênio, clinopiroxênio, plagioclásio, homblenda, biotita, magnetita, ilmenita e pirrotita. Este evento é de natureza sintectônica, com formação de superfícies planares e Kneações minerais. Os minerais analisados são de litologias básicas do complexo.

$\mathrm{O}$ evento metamórfico $\mathrm{M}_{3}$ corresponde à diaftorese relacionada à intrusão dos abundantes corpos graníticos há cerca de $550 \mathrm{Ma}$. Foram formados anfibólios do tipo actinolita, cummingtonita e homblenda, correspondentes a várias fácies do metamorfismo de contato. O ortopiroxênio foi alterado para talco e o clinopiroxênio foi pouco afetado. O plagioclásio foi pouco afetado; em amostras em que foi recristalizado nesse evento, sua composição permanece semelhante ao cristal de $\mathrm{M}_{2}$. Neste evento, não foram geradas superfícies penetrativas e nem lineações minerais. É comum encontrar-se, na mesma amostra, os cristais de minerais máfícos completamente alterados para um número elevado de pequenos cristais de temperatura baixa, sem orientação, enquanto os minerais anidros de $\mathrm{M}_{2}$ permanecem inalterados e com a mesma textura granoblástica de $\mathrm{M}_{2}$. A proximidade e abundância de corpos graníticos intrusivos, mais jovens que o complexo granulítico, e as feições texturais conduzem à hipótese principal de que o evento $\mathrm{M}_{3}$ seja de natureza térmica de contato. 
Em fraturas, ocorrem minerais do tipo epídoto, microclínio e clorita, correspondentes a uni evento que pode ser designado de $\mathrm{M}_{4}$. Esses minerais não foram estudados neste trabalho.

QUÍMICA DE MINERAIS Com o objetivo de investigar a química dos minerais e as condições físicas de sua formação, foram determinadas dezenas de pontos analíticos por dispersão de comprimento de onda. Os minerais analisados são ortopiroxênío, clinopiroxênio, plagioclásio, anfibólio, granada, olivina, espinélios e ilmenita, conforme consta das tabelas 1 a 6 .
O ortopiroxênio tem composição situada entre "hiperstênio" e "bronzita" (Fig. 1). Os clinopiroxênios têm composição mais restrita, correspondendo na maior parte a salita; duas amostras correspondem a diopsídio e uma a diopsídio cálcico (Tabs. 1 e 2)

O plagioclásio tem composição situada entre andesina e anortita (Fig. 2), não tendo sido identificadas composições mais sódicas. O conteúdo de ortoclásio é muito baixo, em geral (Tab. 3).

Os anfíbólios gerados no evento $\mathrm{M}_{2}$ pertencem à série hastingsita-pargasita (Fig. 3). As linhas que unem a compo-

Tabela 1-Análise química (\% peso) e fórmula estrutural de ortopiroxênios

Table 1 - Chemical analysis (weight \%) and structural formula of orthopyroxenes

\begin{tabular}{|c|c|c|c|c|c|c|c|c|c|c|c|c|c|}
\hline \multirow{2}{*}{ CGSMC- } & \multirow[t]{2}{*}{1} & \multicolumn{2}{|c|}{12} & \multirow[t]{2}{*}{17} & \multirow[t]{2}{*}{25} & \multirow[t]{2}{*}{31} & \multirow[t]{2}{*}{35} & \multirow[t]{2}{*}{36} & \multicolumn{2}{|c|}{37} & \multirow[t]{2}{*}{38} & \multirow[t]{2}{*}{39} & \multirow[t]{2}{*}{40} \\
\hline & & $\mathbf{a}$ & $\mathbf{b}$ & & & & & & $\mathbf{a}$ & b & & & \\
\hline $\mathrm{SiO}_{2}$ & 52,38 & 51,39 & 51,55 & 52,26 & 51,22 & 51,17 & 55,04 & 52,44 & 52,30 & 52,37 & 54,90 & 51,65 & 51,72 \\
\hline $\mathrm{TiO}_{2}$ & 0,07 & 0,09 & 0,09 & 0,07 & 0,12 & 0,09 & 0,05 & 0,07 & 0,07 & 0,07 & 0,18 & 0,12 & 0,11 \\
\hline $\mathrm{Al}_{2} \mathrm{O}_{3}$ & 1,35 & 1,02 & 1,04 & 1,26 & 1,87 & 1,24 & 3,03 & 0,99 & 4,01 & 4,21 & 3,11 & 2,46 & 1,29 \\
\hline $\mathrm{Cr}_{2} \mathrm{O}_{3}$ & 0,03 & 0,02 & 0,02 & 0,00 & 0,00 & 0,02 & 0,10 & 0,00 & 0,00 & 0,00 & 0,15 & 0,02 & 0,00 \\
\hline $\mathrm{FeO}_{T}$ & 22,73 & 29,20 & 28,70 & 23,79 & 25,98 & 29,12 & 11,73 & 25,40 & 16,39 & 16,55 & 10,24 & 25,37 & 26,66 \\
\hline $\mathrm{NiO}$ & 0,03 & - & - & 0,00 & - & $=$ & 0,05 & 0,00 & 0,00 & 0,02 & 0,03 & $=$ & - \\
\hline $\mathrm{MnO}$ & 0,48 & 0,68 & 0,65 & 0,66 & 0,67 & 0,62 & 0,30 & 0,73 & 0,39 & 0,36 & 0,25 & 0,35 & 0,69 \\
\hline $\mathrm{CaO}$ & 0,49 & 0,57 & 0,57 & 0,49 & 0,57 & 0,63 & 0,42 & 0,62 & 0,54 & 0,48 & 0,47 & 0,56 & 0,57 \\
\hline $\mathrm{Na}_{2} \mathrm{O}$ & 0,04 & 0,00 & 0,02 & 0,05 & 0,02 & 0,02 & 0,00 & 0,02 & 0,02 & 0,02 & 0,00 & 0,02 & 0,02 \\
\hline \multirow[t]{2}{*}{ Tolal } & 99,63 & 100,38 & 100,19 & 99,65 & 99,64 & 100,08 & 100,75 & 100,32 & 99,68 & 100,00 & 100,09 & 100,86 & 100,83 \\
\hline & \multicolumn{13}{|c|}{ Núnkero de cátionis na base $6(0)$} \\
\hline $\mathbf{S i}$ & 1,9626 & 1,9727 & 1,9771 & 1,9682 & 1,9516 & 1,9698 & 1,9319 & 1,9767 & 1,9002 & 1,8971 & 1,9271 & 1,9336 & 1,9533 \\
\hline $\mathbf{T i}$ & 0,0020 & 0,0025 & 0,0025 & 0,0020 & 0,0035 & 0,0025 & 0,0014 & 0,0020 & 0,0019 & 0,0019 & 0,0048 & 0,0034 & 0,0029 \\
\hline Al & 0,0598 & 0,0460 & 0,0469 & 0,0561 & 0,0840 & 0,0563 & 0,1252 & 0,0441 & 0,1718 & 0,1798 & 0,1287 & 0,1085 & 0,0574 \\
\hline $\mathrm{Cr}$ & 0,0009 & 0,0004 & 0,0004 & 0,0000 & 0,0000 & 0,0004 & 0,0027 & 0,0000 & 0,0000 & 0,0000 & 0,0041 & 0,0004 & 0,0000 \\
\hline $\mathrm{Fe}$ & 0,7122 & 0,9373 & 0,9204 & 0,7494 & 0,8278 & 0,9374 & 0,3442 & 0,8006 & 0,4980 & 0,5014 & 0,3006 & 0,7941 & 0,8419 \\
\hline $\mathrm{Mn}$ & 0,0151 & 0,0222 & 0,0212 & 0,0210 & 0,0217 & 0,0203 & 0,0087 & 0,0234 & 0,0119 & 0,0109 & 0,0074 & 0,0111 & 0,0219 \\
\hline $\mathbf{M g}_{\mathbf{g}}$ & 1,2302 & 0,9963 & 1,0035 & 1,1828 & 1,0897 & 0,9853 & 1,5711 & 1,1264 & 1,4061 & 1,3997 & 1,6097 & 1,1336 & 1,1130 \\
\hline $\mathrm{Ca}$ & 0,0198 & 0,0235 & 0,0235 & 0,0198 & 0,0234 & 0,0259 & 0,0156 & 0,0249 & 0,0210 & 0,0187 & 0,0178 & 0,0225 & 0,0232 \\
\hline $\mathrm{Na}$ & 0,0026 & 0,0000 & 0,0014 & 0,0040 & 0,0013 & 0,0014 & 0,0000 & 0,0013 & 0,0011 & 0,0011 & 0,0000 & 0,0013 & 0,0013 \\
\hline Totst & 4,0061 & 4,0009 & 3,9969 & 4,0033 & 4,0030 & 3,9993 & 4,0020 & 3,9994 & 4,0120 & 4,0110 & 4,0010 & 4,0085 & 4,0149 \\
\hline $\mathrm{Mg}$ & 62,2 & 50,3 & 51,0 & 60,0 & 55,5 & 50,1 & 81,0 & 57,0 & 72,6 & 72,5 & 83,2 & 57,8 & 55,6 \\
\hline $\mathrm{Fe}+\mathrm{Mn}$ & 36,8 & 48,5 & 47,8 & 39,0 & 43,3 & 48,6 & 18,2 & 41,7 & 26,3 & 26,5 & 15,9 & 41,1 & 43,2 \\
\hline $\mathrm{Ca}$ & 1,0 & 1,2 & 1,2 & 1,0 & 1,2 & 1,3 & 0,8 & 1,3 & 1,1 & 1,0 & 0,9 & 1,1 & 1,2 \\
\hline
\end{tabular}

Tabela 2 -Análise química (\% peso) e fórmula estrutural de clinopiroxêuios

Table 2 - Chemical analysis (weight \%) and structural formula of clinopyroxenes

\begin{tabular}{|c|c|c|c|c|c|c|c|c|c|c|c|c|c|c|}
\hline \multirow{2}{*}{ CGSMC- } & 1 & 12 & 17 & 25 & 31 & 34 & 35 & 36 & \multicolumn{3}{|c|}{37} & \multirow[t]{2}{*}{38} & \multirow[t]{2}{*}{39} & \multirow[t]{2}{*}{40} \\
\hline & & & & & & & & & $\mathbf{a}$ & b & c & & & \\
\hline $\mathrm{SiO}_{2}$ & 51,52 & 52,02 & 52,53 & 50,77 & 50,95 & 51,07 & 53,23 & 52,03 & 49,68 & 49,75 & 49,33 & 50,73 & 49,51 & 51,82 \\
\hline $\mathrm{TiO}_{2}$ & 0,28 & 0,21 & 0,17 & 0,41 & 0,30 & 0,39 & 0,21 & 0,22 & 0,45 & 0,51 & 0,49 & 0,70 & 0,60 & 0,24 \\
\hline $\mathrm{Al}_{2} \mathrm{O}_{3}$ & 2,43 & 1,82 & 1,77 & 3,46 & 2,42 & 3,55 & 3,18 & 1,92 & 6,01 & 6,21 & 6,62 & 3,96 & 4,64 & 2,23 \\
\hline $\mathrm{Cr}_{2} \mathrm{O}_{3}$ & 0,10 & 0,05 & 0,00 & 0,03 & 0,03 & 0,00 & 0,16 & 0,00 & 0,00 & 0,00 & 0,02 & 0,26 & 0,05 & 0,02 \\
\hline $\mathrm{FeO}_{\mathrm{T}}$ & 8,60 & 11,14 & 9,20 & 10,02 & 11,86 & 10,71 & 4,27 & 10,16 & 6,18 & 6,25 & 6,11 & 4,25 & 10,88 & 10,60 \\
\hline NiO & 0,03 & - & 0,00 & - & - & - & 0,03 & 0,00 & 0,00 & 0,02 & 0,00 & - & $=$ & $=$ \\
\hline $\mathrm{M}: \mathrm{O}$ & 0,20 & 0,27 & 0,23 & 0,28 & 0,25 & 0,13 & 0,14 & 0,31 & 0,13 & 0,19 & 0,11 & 0,13 & 0,17 & $0,3 \mathrm{~J}$ \\
\hline $\mathrm{CaO}$ & 22,36 & 22,14 & 22,49 & 21,93 & 21,23 & 22,04 & 24,08 & 22,78 & 23,82 & 23,79 & 23,85 & 23,64 & 21,34 & 21,75 \\
\hline $\mathrm{Na}_{2} \mathrm{O}$ & 0,48 & 0,48 & 0,62 & 0,50 & 0,48 & 0,57 & 0,20 & 0,54 & 0,52 & 0,47 & 0,50 & 0,17 & 0,74 & 0,62 \\
\hline \multirow[t]{2}{*}{ Total } & 99,33 & 100,16 & 99,69 & 99,47 & 99,12 & 100,63 & 101,17 & 100,61 & 100,20 & 100,51 & 100,19 & 99,34 & 100,22 & 100,13 \\
\hline & \multicolumn{14}{|c|}{ Nútrero de éflions un base $6(0)$} \\
\hline$\overline{\mathbf{S i}}$ & 1,9372 & 1,9595 & 1,9612 & 1,9168 & 1,9433 & 1,9106 & 1,9278 & 1,9470 & $1,8+04$ & 1,8376 & 1,8271 & 1,8777 & 1,8770 & 1,9468 \\
\hline $\mathrm{Ti}$ & 0,0079 & 0,0058 & 0,0047 & 0,0116 & 0,0085 & 0,0110 & 0,0056 & 0,0063 & 0,0126 & 0,0141 & 0,1036 & 0,0195 & 0,0168 & 0,0068 \\
\hline Al & 0,1077 & 0,0807 & 0,0784 & 0,1538 & 0,1088 & 0,1564 & 0,1358 & 0,0847 & 0,2623 & 0,2702 & 0,2892 & 0,1729 & 0,2057 & 0,0989 \\
\hline $\mathbf{C r}$ & 0,0030 & 0,0015 & 0,0000 & 0,0010 & 0,0010 & 0,0000 & 0,0044 & 0,0000 & 0,0000 & 0,0000 & 0,0005 & 0,0076 & 0,0015 & 0,0005 \\
\hline Mir & 0,0064 & 0,0084 & 0,6074 & 0,0090 & 0,0080 & 0,0039 & $0,00+3$ & 0,0099 & 0,0039 & 0,0059 & 0,0034 & 0,0039 & $0,00 \$ 4$ & 0,0099 \\
\hline $\mathrm{Mg}_{\mathrm{g}}$ & 0,7471 & 0,6756 & 0,7265 & 0,6795 & 0,6954 & 0,6786 & 0,8458 & 0,7053 & 0,7407 & 0,7333 & 0,7266 & 0,8552 & 0,6663 & 0,7020 \\
\hline $\mathrm{Ca}$ & 0,9008 & 0,8935 & 0,9049 & 0,8872 & 0,8676 & 0,8835 & 0,9346 & 0,9134 & 0,9455 & 0,9414 & 0,9463 & 0,9376 & 0,8598 & 0,8756 \\
\hline $\mathrm{Na}$ & 0,0350 & 0,0347 & 0,0448 & 0,0369 & 0,0355 & 0,0415 & 0,0143 & 0,390 & 0,0370 & 0,0334 & 0,0358 & 0,0125 & 0,0542 & 0,0454 \\
\hline Total & 4,0164 & 4,0105 & 4,0166 & 4,0121 & 4,0105 & 4,0205 & 4,0028 & 4,0235 & 4,0339 & 4,0294 & 4,0318 & 4,0183 & 4,0290 & 4,0189 \\
\hline $\mathbf{M g}$ & 38,8 & 35,0 & 37,7 & 35,9 & 35,7 & 35,7 & 44,2 & 36,2 & 39,4 & 39,1 & 39,0 & 44,4 & 35,6 & 36,6 \\
\hline $\mathrm{Fe}+\mathrm{Mm}$ & 14,4 & 18,6 & 15,4 & 17,2 & 19,8 & 17,8 & 7,0 & 16,9 & 10,4 & 10,6 & 10,3 & 7,0 & 18,5 & 17,8 \\
\hline $\mathrm{Ca}$ & 46,8 & 46,4 & 46,9 & 46,9 & 44,5 & 46,5 & 48,8 & 46,9 & 50,2 & 50,2 & 50,7 & 48,6 & 45,9 & 45,6 \\
\hline
\end{tabular}


Tabela 3 -Análise química (\% peso) e fórmula estrutural de plagioclásios

Table 3 - Chemical analysis (weight $\%$ ) and slructural formula of plagioclase

\begin{tabular}{|c|c|c|c|c|c|c|c|c|c|c|c|c|c|c|c|c|c|c|c|c|}
\hline \multirow{2}{*}{ CGSMC. } & \multirow[t]{2}{*}{1} & \multicolumn{3}{|c|}{3} & \multirow[t]{2}{*}{12} & \multirow[t]{2}{*}{17} & \multirow[t]{2}{*}{25} & \multirow[t]{2}{*}{31} & \multicolumn{2}{|c|}{32} & \multicolumn{3}{|c|}{33} & \multirow[t]{2}{*}{34} & \multirow[t]{2}{*}{36} & \multicolumn{2}{|c|}{37} & \multirow[t]{2}{*}{39} & \multicolumn{2}{|c|}{40} \\
\hline & & a & b & c & & & & & a & b & a & b & c & & & a & b & & a & b \\
\hline $\mathrm{SiO}_{2}$ & 55,29 & 46,58 & 45,48 & 46,89 & 51,31 & 61,14 & 52,31 & 55,65 & 49,15 & 50,61 & 60,53 & 60,32 & 60,00 & 55,31 & 58,43 & 46,14 & 44,38 & 55,98 & 57,23 & 57,83 \\
\hline $\mathrm{TiO}_{2}$ & 0,02 & - & - & - & - & 0,04 & - & - & 0,02 & 0,02 & 0,02 & 0,04 & 0,02 & 0,02 & 0,02 & 0,02 & 0,02 & 0,02 & 0,02 & 0,02 \\
\hline $\mathrm{Al}_{2} \mathrm{O}_{3}$ & 27,77 & 33,83 & 34,75 & 33,54 & 26,03 & 24,60 & 29,63 & 27,69 & 31,90 & 31,32 & 24,69 & 24,35 & 24,54 & 27,97 & 26,29 & 34,25 & 35,73 & 28,12 & 26,74 & 26,47 \\
\hline $\mathrm{reOr}$ & 0,13 & 0,07 & 0,08 & 0,10 & 0,11 & 0,14 & 0,13 & 0,11 & 0,20 & 0,04 & 0,03 & 0,01 & 0,01 & 0,15 & 0,20 & 0,17 & 0,00 & 0,08 & 0,25 & 0,13 \\
\hline $\mathrm{CaO}$ & 10,26 & 17,64 & 18,53 & 17,31 & 9,04 & 6,45 & 12,70 & 10,30 & 15,13 & 14,60 & 6,23 & 6,14 & 6,28 & 10,72 & 8,51 & 18,06 & 19,87 & 10,41 & 8,76 & 8,53 \\
\hline $\mathrm{Na}_{2} \mathrm{O}$ & 5,45 & 1,57 & 1,09 & 1,69 & 6,53 & 7,58 & 4,30 & 5,60 & 3,03 & 3,57 & 7,98 & 7,98 & 7,89 & 5,50 & 6,71 & 1,44 & 0,65 & 5,78 & 6,67 & 6,80 \\
\hline $\mathrm{K}_{2} \mathrm{O}$ & 0,32 & 0,01 & 0,00 & 0,00 & 0,05 & 0,50 & 0,08 & 0,16 & 0,01 & 0,01 & 0,38 & 0,36 & 0,42 & 0,31 & 0,31 & 0,00 & 0,00 & 0,15 & 0,18 & 0,20 \\
\hline Tolal & \multicolumn{20}{|c|}{ Número de cátions ma base $8(0)$} \\
\hline$\overline{\mathrm{Si}}$ & 2,5089 & 2,1484 & 2,0985 & 2,1639 & $2,593 \overline{3}$ & 2,7093 & 2,3959 & 2,5151 & 2,2590 & 2,3034 & 2,6987 & 2,7063 & 2,6955 & 2,4963 & 2,6062 & 2,1243 & 2,0420 & 2,5070 & 2,5707 & 2,5923 \\
\hline$\pi$ & 0,0006 & & & & & 0,0012 & & & 0,0006 & 0,0006 & 0,0006 & 0,0012 & 0,0006 & 0,0006 & 0,0006 & 0,0006 & 0,0006 & 0,0006 & 0,0006 & 0,0006 \\
\hline $\mathbf{A l}$ & 1,4853 & 1,8389 & 1,8901 & 1,8246 & 1,3884 & 1,2851 & 1,5930 & 1,4752 & 1,7284 & 1,6801 & 1,2977 & 1,2876 & 1,2996 & 1,4880 & 1,3823 & 1,8588 & 1,9375 & 1,4846 & 1,4159 & 1,3989 \\
\hline $\mathrm{Fe}$ & 0,0048 & 0,0027 & 0,0032 & 0,0038 & 0,0042 & 0,0052 & 0,0048 & 0,0042 & 0,0075 & 0,0016 & 0,0010 & 0,0005 & 0,0005 & 0,0058 & 0,0073 & 0,0065 & 0,0000 & 0,0031 & 0,0095 & 0,0047 \\
\hline $\mathrm{Ca}$ & 0,4990 & 0,8716 & 0,9160 & 0,8560 & 0,4384 & 0,3064 & 0,6207 & 0,4990 & 0,7452 & 0,7121 & 0,2978 & 0,2954 & 0,3020 & 0,5186 & 0,4065 & 0,8909 & 0,9795 & 0,4995 & 0,4214 & 0,4097 \\
\hline $\mathrm{Na}$ & 0,4796 & 0,1402 & 0,0973 & 0,1508 & 0,5730 & 0,6509 & 0,3802 & 0,4979 & 0,2698 & 0,3152 & 0,6900 & 0,6944 & 0,6872 & 0,4816 & 0,5805 & 0,1284 & 0,0577 & 0,5017 & 0,5812 & 0,5908 \\
\hline $\mathbf{K}$ & 0183 & 0,0006 & 0,0000 & 0,0000 & 0,0027 & 0,0282 & 0,0047 & 0,0095 & 0,0006 & 0,0006 & 0,0216 & 0,0204 & 0,0238 & 0,0176 & 0,0174 & 0,0000 & 0,0000 & 0,0087 & 0,0101 & 0,0114 \\
\hline Tolal & 4,9965 & 5,0024 & 5,0051 & 4,9991 & 5,0000 & 4,9863 & 4,997 & 5,0009 & 5,0111 & 5,0136 & 5,0074 & 5,0058 & 5,0092 & 5,0085 & 5,0008 & 5,0095 & 5,0173 & 5,0052 & 5,0094 & 5,0084 \\
\hline$A b$ & 46,6 & 13,2 & 9,0 & 14,2 & 55,0 & 64,7 & 36,4 & 48,0 & 25,4 & 29,4 & 67,1 & 67,4 & 66,5 & 45,8 & 56,3 & 12,0 & 5,3 & 48,2 & 55,9 & 56,9 \\
\hline Or & 1,9 & 0,1 & 0,0 & 0,0 & 0,0 & 3,0 & 0,5 & 1,0 & 0,1 & 0,1 & 2,2 & 2,2 & 2,5 & 1,8 & 1,8 & 0,0 & 0,0 & 0,9 & 1,1 & 1,2 \\
\hline
\end{tabular}

sição dos anfibólios aos piroxênios na figura 1 são aproximadamente paralelas, indicando condições próximas ao equilíbrio. Esta conclusão coincide com as observações texturais. $\mathrm{Na}$ figura 3, são também mostradas as composições dos anfibólios cálcicos do evento térmico $\mathrm{M}_{3}$. As flechas indicam a variação composicional do anfibólio de $\mathrm{M}_{2}$ para o anfibólio de Ma, na mesma amostra. Uma amostra indica que o anfibólio diaftorético corresponde a hornblenda, gerada a partir de uma homblenda de alto grau; esta última teve sua composição parcialmente afetada pela formação do novo mineral, conforme visto ao microscópio e mostrado na figura 3 . Os dados constam da tabela 4.

As granadas analisadas são soluções sólidas almandina-piropo ricas em grossulária (Fig. 4). A zonação dos cristais não é muito acentuada, e corresponde a núcleos mais ricos em
$\mathrm{Mg}$, passando a bordos mais ricos em $\mathrm{Fe}$. As análises constam da tabela 5.

Os espinélios analisados correspondem a magnetita e hercinita, tendo sido analisada ainda ilmenita (Tab. 6).

$\mathrm{Na}$ amostra CGSMC-38, foram analisados seis pontos em dois cristais de olivina, fornecendo valores médios de $\mathrm{SiO}_{2}$ em tomo de $40 \%, \mathrm{Cr}_{2} \mathrm{O}_{3}$ em tomo de $0 \%, \mathrm{CaO}$ em torno de $0,03 \%$ e $\mathrm{Al}_{2} \mathrm{O}_{3}$ em tomo de $0 \%$. O conteúdo extremamente baixo dos três elementos-traços indica cristalização metamórfica para a olivina deste espinélio lherzolito.

GEOBAROMETRIA No artigo de Hartmann (1988), foram apresentados os resultados barométricos nas duas amostras estudadas com mais detalhe. As amostras CGSMC-37 e -39 foram selecionadas para estudo por apresentarem as pa-

Tabela 4 -Análise química (\% peso) e fórmula estrutural de anfibólios

Table 4 - Chemical analysis (weight $\%$ ) and structural formula of amphiboles

\begin{tabular}{|c|c|c|c|c|c|c|c|c|c|c|c|}
\hline \multirow{2}{*}{ COSMC- } & \multicolumn{4}{|c|}{1} & \multicolumn{2}{|c|}{3} & \multicolumn{3}{|c|}{12} & \multicolumn{2}{|c|}{25} \\
\hline & $\mathbf{a}$ & $\mathbf{b}$ & $\mathrm{c}$ & d & $a$ & $b$ & $a$ & b & $\mathrm{c}$ & a & $b$ \\
\hline $\mathrm{SiO}_{2}$ & 43,23 & 54,05 & 52,77 & 52,47 & 45,08 & 45,01 & 43,08 & 53,29 & 49,65 & 42,97 & 51,33 \\
\hline $\mathrm{TO}_{2}$ & 1,76 & 0,04 & 0,11 & 0,06 & 0,09 & 0,07 & 1,74 & 0,07 & 0,36 & 1,59 & 0,22 \\
\hline $\mathrm{Al}_{2} \mathrm{O}_{3}$ & 11,17 & 2,43 & 3,72 & 3,81 & 13,29 & 13,29 & 10,74 & 1,62 & 4,86 & 11,71 & 3,98 \\
\hline $\mathrm{Cr}_{2} \mathrm{O}_{3}$ & 0,23 & 0,02 & 0,00 & 0,02 & - & - & - & - & - & - & - \\
\hline $\mathrm{FrOO}_{\mathrm{r}}$ & 13,07 & 15,93 & 15,25 & 13,02 & 10,73 & 11,24 & 16,83 & 16,51 & 17,17 & 15,52 & 17,22 \\
\hline Mno & 0,12 & 0,51 & 0,39 & 0,34 & 0,20 & 0,23 & 0,20 & 0,43 & 0,31 & 0,19 & 0,46 \\
\hline $\mathrm{MgO}$ & 12,58 & 17,19 & 16,25 & 16,20 & 13,70 & 13,56 & 10,06 & 14,34 & 11,93 & 11,05 & 17,09 \\
\hline $\mathrm{CaO}$ & 11,81 & 6,98 & 8,17 & 10,75 & 16,75 & 11,79 & 11,38 & 10,60 & 11,82 & 11,78 & 5,33 \\
\hline $\mathrm{Na}_{2} \mathrm{O}$ & 1,26 & 0,17 & 0,33 & 0,42 & 1,64 & 1,63 & 1,63 & 0,16 & 0,57 & 1,81 & 0,56 \\
\hline $\mathrm{K}_{2} \mathrm{O}$ & 1,50 & 0,01 & 0,09 & 0,05 & 0,09 & 0,10 & 0,58 & 0,02 & 0,15 & 0,73 & 0,26 \\
\hline \multirow[t]{2}{*}{ Total } & 96,73 & 97,33 & 97,08 & 97,14 & 96,57 & 96,92 & 96,24 & 97,04 & 96,72 & 97,35 & 96,45 \\
\hline & \multicolumn{11}{|c|}{ Nünero de cátions na base $23(0)$} \\
\hline $\mathbf{S i}$ & $6,4,62$ & 7,7937 & 7,6477 & 7,5859 & 6,5787 & 6,5638 & 7,8249 & 7,8249 & 7,4024 & 6,4395 & 7,5338 \\
\hline Ti & 0,1980 & 0,0039 & 0,0119 & 0,0059 & 0,0101 & 0,0080 & 0,0080 & 0,0080 & 0,0409 & 0,1788 & 0,0239 \\
\hline Al & 1,9693 & 0,4129 & 0,6347 & 0,6498 & 2,2864 & 2,2841 & 0,2800 & 0,2800 & 0,8558 & 2,0687 & 0,6890 \\
\hline Cr & 0,0277 & 0,0018 & 0,0000 & 0,0019 & & & & & & & \\
\hline $\mathrm{Fe}^{3+}$ & 0,4114 & & & 1,2125 & 0,6620 & 0,7088 & 0,4555 & 1,1510 & 0,4887 & 0,4328 & \\
\hline $\mathrm{Fe}^{2 *}$ & 1,2092 & & & 0,3205 & 0,6289 & 0,6410 & 1,6051 & 0,8260 & 1,8328 & 1,4939 & \\
\hline $\mathrm{Fe}$ & & 1,9208 & 1,8479 & & & & & & & & 2,1136 \\
\hline Mn & 0,0157 & 0,0624 & 0,0475 & 0,0419 & 0,0250 & 0,0288 & 0,0259 & 0,0539 & 0,0391 & 0,0235 & 0,0576 \\
\hline $\mathbf{M g}$ & 2,8060 & 3,6953 & 3,5100 & 3,4919 & 2,9794 & 2,9480 & 2,2816 & 3,1389 & 2,6569 & 2,4679 & $\mathbf{3 , 7 3 7 8}$ \\
\hline $\mathrm{Ca}$ & 1,8933 & 1,0783 & 1,2681 & 1,6659 & 1,8373 & 1,8415 & 1,8554 & 1,6681 & 1,8919 & 1,8908 & 0,8380 \\
\hline $\mathrm{Na}$ & 0,3658 & 0,0482 & 0,0918 & 0,1183 & 0,4644 & 0,4617 & 0,4812 & 0,0451 & 0,1648 & 0,5261 & 0,1588 \\
\hline $\mathrm{K}$ & 0,2858 & 0,0021 & 0,0171 & 0,0085 & 0,0171 & 0,0192 & 0,1128 & 0,0043 & 0,0283 & 0,1396 & 0,0478 \\
\hline Total & 15,6625 & {$[5,0194$} & 15,0767 & 15,1448 & 15,4893 & 15,5049 & 14,9904 & 15,0002 & 15,4016 & 15,6616 & 15,2003 \\
\hline
\end{tabular}


Tabela 4 - -Continuação

Table 4 - Continuing

\begin{tabular}{|c|c|c|c|c|c|c|c|c|c|c|c|c|c|}
\hline \multirow{2}{*}{ CGSMC- } & \multicolumn{3}{|c|}{31} & \multicolumn{3}{|c|}{32} & \multirow[t]{2}{*}{34} & \multirow[t]{2}{*}{35} & \multirow[t]{2}{*}{36} & \multicolumn{2}{|c|}{37} & \multirow[t]{2}{*}{38} & \multirow[t]{2}{*}{39} \\
\hline & a & $\mathrm{b}$ & c & a & $\mathrm{b}$ & $c$ & & & & $\mathfrak{a}$ & b & & \\
\hline $\mathrm{SiO}_{2}$ & 42,12 & 53,42 & 54,10 & 44,84 & 47,03 & 48,42 & 40,63 & 45,00 & 43,39 & 41,56 & 41,83 & 43,03 & 41,58 \\
\hline $\mathrm{TjO}_{2}$ & 2,07 & 0,11 & 0,09 & 0,48 & 0,39 & 0,20 & 2,68 & 0,74 & 1,81 & 1,48 & 1,49 & 1,49 & 2,25 \\
\hline $\mathrm{Al}_{2} \mathrm{O}_{3}$ & 11,76 & 1,86 & 1,39 & $12,62$. & 10,71 & 9,16 & 12,73 & 13,20 & 11,17 & 15,31 & 15,10 & 12,85 & 13,29 \\
\hline $\mathrm{Cr}_{2} \mathrm{O}_{3}$ & - & - & - & 0,12 & 0,03 & 0,00 & - & 0,39 & 0,00 & 0,00 & 0,03 & - & - \\
\hline FeOT & 17,13 & 13,50 & 13,80 & 13,37 & 12,55 & 12,04 & 16,62 & 7,20 & 14,93 & 10,08 & 9,89 & 6,30 & 13,59 \\
\hline MnO & 0,17 & 0,20 & 0,23 & 0,20 & 0,20 & 0,20 & 0,06 & 0,06 & 0,15 & 0,14 & 0,11 & 0,09 & 0,08 \\
\hline $\mathrm{MgO}$ & 9,66 & 14,98 & 14,98 & 12,28 & 13,30 & 14,33 & 9,86 & 16,30 & 11,78 & 13,61 & 13,93 & 16,47 & 11,89 \\
\hline $\mathrm{CaO}$ & 11,67 & 12,53 & 12,58 & 11,96 & 12,13 & 12,11 & 11,77 & 12,50 & 12,16 & 12,36 & 12,25 & 12,82 & 11,78 \\
\hline $\mathrm{Na}_{2} \mathrm{O}$ & 1,53 & 0,14 & 0,12 & 1,22 & 1,02 & 0,92 & 1,56 & 2,02 & 1,27 & 2,63 & 2,68 & 1,90 & 2,35 \\
\hline $\mathrm{K}_{2} \mathrm{O}$ & 1,44 & 0,10 & 0,03 & 0,32 & 0,23 & 0,14 & 1,92 & 0,45 & 1,24 & 0,23 & 0,26 & 0,86 & 0,91 \\
\hline \multirow{2}{*}{ Total } & $\overline{97,55}$ & 96,84 & 97,32 & 97,41 & 97,59 & 97,52 & 97,83 & 97,95 & 97,90 & 97,40 & 97,57 & 95,81 & 97,72 \\
\hline & \multicolumn{13}{|c|}{ Número de cátions na base 23 (0) } \\
\hline$\overline{\mathrm{Si}}$ & 6,3760 & 7,7905 & 7,8613 & 6,5813 & 6,8351 & 7,0094 & 6,1564 & 6,3652 & 6,4601 & 6,0819 & 6,1020 & 6,3019 & 6,1821 \\
\hline Ti & 0,2359 & 0,0121 & 0,0100 & 0,0526 & 0,0422 & 0,0220 & 0,3048 & 0,0785 & 0,2026 & 0,1624 & 0,1640 & 0,1640 & 0,2517 \\
\hline Al & 2,0983 & 0,3191 & 0,2383 & 2,1830 & 1,8352 & 1,5621 & 2,2744 & 2,2015 & 1,9608 & 2,6406 & 2,5958 & 2,2178 & 2,3286 \\
\hline Cr & & & & 0,0135 & 0,0038 & 0,0000 & & 0,0435 & 0,0000 & 0,0000 & 0,0039 & & \\
\hline $\mathrm{Fe}^{3 *}$ & 0,2865 & 0,1762 & 0,1524 & 0,6389 & 0,5183 & 0,5847 & 0,2667 & 0,4410 & 0,4055 & 0,3629 & 0,4052 & 0,2203 & 0,3474 \\
\hline $\mathrm{Fe}^{2+}$ & 1,8688 & 0,4641 & 1,5171 & 0,9794 & 0,9901 & 0,8543 & 1,8272 & 0,4165 & 1,4373 & 0,8608 & 0,7909 & 0,5477 & 1,3299 \\
\hline $\mathrm{Fe}$ & & & & & & & & & & & & & \\
\hline Mn & 0,0217 & 0,0249 & 0,0286 & 0,0250 & 0,0248 & 0,0248 & 0,0079 & 0,0070 & 0,0195 & 0,0173 & 0,0134 & 0,0116 & 0,0097 \\
\hline Mg & 2,1805 & 3,2576 & 3,2395 & 2,6864 & 2,8811 & 3,0911 & 2,2263 & 3,4410 & 2,6136 & 2,9680 & 3,0296 & 3,5962 & 2,6363 \\
\hline $\mathrm{Ca}$ & 1,8929 & 1,9582 & 1,9567 & 1,8803 & 1,8897 & 1,8779 & 1,9113 & 1,8921 & 1,9398 & 1,9375 & 1,9150 & 2,0113 & 1,8772 \\
\hline $\mathrm{Na}$ & 0,4485 & 0,0386 & 0,0337 & 0,3474 & 0,2888 & 0,2577 & 0,4571 & 0,5524 & 0,3651 & 0,7449 & 0,7587 & 0,5403 & 0,6773 \\
\hline$K$ & 0,2790 & 0,0192 & 0,0063 & 0,0601 & $0,0+26$ & 0,0255 & 0,3711 & 0,0810 & 0,2362 & 0,0428 & 0,0491 & 0,1613 & 0,1734 \\
\hline Total & 15,6881 & 14,0605 & 15,0339 & 15,4710 & 15,3682 & 15,3285 & 15,8149 & 15,5300 & 15,6569 & 15,8286 & 15,8376 & 15,7754 & 15,8267 \\
\hline
\end{tabular}

Tabela 5 -Análise química (\% peso) e fórmula estrutural de granadas

Table 5 - Chemical analysis (weight \%) and struclural formula of gameis

\begin{tabular}{|c|c|c|c|c|c|c|c|c|}
\hline \multirow{2}{*}{ CGSNic- } & \multicolumn{3}{|c|}{33} & \multicolumn{2}{|c|}{34} & \multicolumn{2}{|c|}{37} & \multirow[t]{2}{*}{39} \\
\hline & $\mathrm{a}$ & b & $\mathrm{c}$ & $a$ & $\bar{b}$ & $\mathrm{a}$ & $\bar{b}$ & \\
\hline $\mathrm{SiO}_{2}$ & 39,59 & 39,90 & 39,53 & 39,04 & 39,10 & 40,77 & 40,14 & 39,41 \\
\hline $\mathrm{TiO}_{2}$ & 0,04 & 0,05 & 0,07 & 0,09 & 0,07 & 0,05 & 0,04 & 0,11 \\
\hline $\mathrm{Al}_{2} \mathrm{O}_{3}$ & 22,50 & 22,59 & 22,58 & 21,55 & 21,56 & 22,80 & 22,63 & 21,91 \\
\hline $\mathrm{Cr}_{2} \mathrm{O}_{3}$ & 0,08 & 0,06 & 0,06 & $0, \infty$ & 0,00 & 0,00 & 0,00 & 0,02 \\
\hline FeoT & 26,60 & 26,45 & 26,43 & 27,58 & 27,94 & 18,24 & 19,42 & 24,55 \\
\hline M॥O & 0,37 & 0,35 & 0,38 & 0,85 & 0,97 & 0,79 & 1,21 & 0,82 \\
\hline $\mathrm{MgO}$ & 10,94 & 11,03 & 11,09 & 6,06 & 5,67 & 11,79 & 10,40 & 7,55 \\
\hline $\mathrm{CaO}$ & 1,13 & 1,13 & 1,10 & 6,66 & 6,70 & 7,32 & 7,40 & 7,29 \\
\hline \multirow[t]{2}{*}{ Tolal } & 101,13 & 101,56 & 101,24 & 101,83 & 102,01 & 101,79 & 101,26 & 101,66 \\
\hline & \multicolumn{8}{|c|}{ Núnero de cátions la base 24 (0) } \\
\hline$\overline{\mathrm{Si}}$ & 5,9689 & 5,9864 & 5,9553 & 5,9954 & 6,0058 & 5,9920 & 5,9814 & 5,9850 \\
\hline Ti & 0,0039 & 0,0059 & 0,0079 & 0,0101 & 0,0080 & 0,0058 & 0,0040 & 0,0121 \\
\hline Al & 3,9981 & 3,9944 & 4,0104 & 3,9012 & 3,9032 & 3,9492 & 3,9740 & 3,9224 \\
\hline Cr & 0,0092 & 0,0073 & 0,0074 & 0,0000 & 0,0000 & 0,0000 & 0,0000 & 0,0019 \\
\hline $\mathrm{Fe}$ & 3,3541 & 3,3195 & 3,3298 & 3,5427 & 3,5888 & 2,2420 & 2,4204 & 3,1179 \\
\hline$M_{11}$ & 0,0466 & 0,0445 & 0,0486 & 0,1108 & 0,1264 & 0,0976 & 0,1532 & 0,1061 \\
\hline $\mathrm{Mg}$ & 2,4590 & 2,4661 & 2,4897 & 1,3865 & 1,2988 & 2,5820 & 2,3102 & 1,7086 \\
\hline $\mathrm{Ca}_{\mathrm{a}}$ & 0,1829 & 0,1821 & 0,1783 & 1,0968 & 1,1028 & 1,1528 & 1,1810 & 1,1863 \\
\hline \multirow[t]{2}{*}{ Total } & 16,0227 & 16,0062 & 16,0274 & 16,0435 & 16,0376 & 16,0312 & 16,0292 & 16,0403 \\
\hline & nícleo & intermediário & borda & & & núcleo & borda & \\
\hline
\end{tabular}

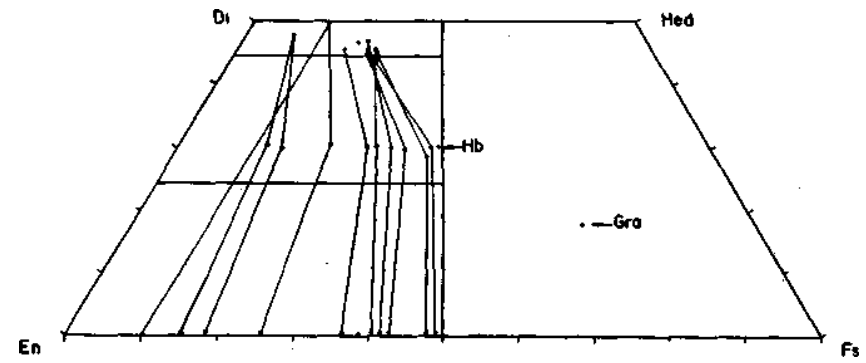

Figura 1 - Composição de pi roxéi lios, hornblenda e granada Figure l - Composilion of pyroxenes, hornblende and garnet

ragêneses e texturas mais adequadas para estudos barométricos. A pressão obtida é da ordem de 9,4 kbar para o evento $\mathrm{M}_{2}$. Na amostra -37 , foram analisados ainda cristais pequenos de granada, clinopiroxênio e plagioclásio, sendo obtida uma pressão de $8,4 \mathrm{kbar}$, para uma temperatura estipulada era $700^{\circ} \mathrm{C}$. Nos mesmos cristais, obtém-se uma pressão de 8,1 kbar, ao serem empregados granada, ortopiroxênio e plagioclásio. Este evento granulítico de pressão mais baixa, em torno de 8,2 kbar, somente foi observado nesta amostra, e é considerado como um reajuste local a condições declinantes de metamorfismo. A amostra parece corresponder a um metassedimento, pois os teores de $\mathrm{Cr}$ e Ni são muito baixos, tanto na amostra quanto nos minerais máficos.

As moléculas de jadeíta e tschermakita foram calculadas pelo método de Cawthorn \& Collerson (1974) em clinopiroxênio de duas amostras. Estas moléculas são geralmente consideradas como bons indicadores de condições de pressão. Os valores obtidos na amostra CGSMC-34 são de 4,1 para jadeíta e 4,6 para tschermakita, e na amostra -39 de 5,4 e 5,8. Estes valores são altos, indicando condições de alta pressão para a 
Tabela 6 - Análises químicas (\% peso) e fórmula estrutural de espinélio e ilmenita: mag = magnetita; ilm = ilmenita; her = hercynita; pie = "picotita" (= espinélio com $\mathrm{Cr}$ )

Table 6 - Chemical analysis (weight \%) and structural formula of spinels and ilmenite: mag - magnetite; ilm - ilmenite; her - hercynite; pie - "picotite" (çromiai. spinel)

\begin{tabular}{|c|c|c|c|c|c|c|c|c|c|c|c|}
\hline \multirow{2}{*}{ COSSMC- } & \multicolumn{2}{|c|}{17} & \multirow[t]{2}{*}{34} & \multirow[t]{2}{*}{35} & \multirow[t]{2}{*}{36} & \multicolumn{2}{|c|}{37} & \multicolumn{2}{|c|}{38} & \multirow[t]{2}{*}{39} & \multirow[t]{2}{*}{40} \\
\hline & 2 & b & & & & 3 & $b$ & $\mathbf{a}$ & $\bar{b}$ & & \\
\hline $\mathrm{SiO}_{2}$ & 0,11 & 0,05 & 2,64 & 0,13 & 0,07 & 0,06 & 0,06 & 0,06 & 0,06 & 0,07 & 0,34 \\
\hline $\mathrm{TO}_{2}$ & 0,21 & 46,77 & 52,61 & 0,16 & 49,71 & 0,03 & 0,02 & 0,07 & 0,08 & 51,04 & 49,84 \\
\hline $\mathrm{Al}_{2} \mathrm{O}_{3}$ & 0,16 & 0,00 & 0,18 & 1,47 & 0,02 & 62,63 & 63,29 & 48,69 & 48,92 & 0,02 & 0,07 \\
\hline $\mathrm{C}_{2} \mathrm{O}_{3}$ & 0,10 & 0,00 & 0,02 & 7,12 & 0,02 & 0,05 & 0,06 & 12,84 & 12,09 & 0,03 & 0,00 \\
\hline $\mathrm{FeO}_{r}$ & 91,51 & 49,48 & 40,02 & 83,87 & 48,83 & 25,61 & 24,04 & 24,15 & 25,15 & 46,98 & 46,82 \\
\hline $\mathrm{NiO}$ & - & $=$ & - & $=$ & $=$ & 0,09 & 0,09 & - & - & - & - \\
\hline $\mathrm{MnO}$ & 0,03 & 1,82 & 1,46 & 0,39 & 0,58 & 0,18 & 0,20 & 0,22 & 0,24 & 0,29 & 1,26 \\
\hline $\mathrm{CaO}$ & 0,00 & 0,04 & 2,68 & 0,04 & 0,02 & 0,01 & 0,04 & 0,01 & 0,01 & 0,02 & 0,02 \\
\hline \multirow[t]{2}{*}{ Tolal } & 92,14 & 98,16 & 99,61 & 93,58 & 100,17 & 101,70 & 101,14 & 100,24 & 100,39 & 99,79 & 98,69 \\
\hline & \multicolumn{11}{|c|}{ Núneno de cátions na base $32(0)$} \\
\hline$\overline{\mathrm{Si}}$ & 0,0438 & 0,0139 & 0,6917 & 0,0512 & 0,0201 & 0,0128 & 0,0128 & 0,0131 & 0,0132 & 0,0199 & 0,0946 \\
\hline $\mathrm{Ti}$ & 0,0613 & 9,9244 & 10,3484 & 0,0478 & 10,1624 & 0,0055 & 0,0027 & 0,0113 & 0,0142 & 10,3596 & 10,2929 \\
\hline $\mathrm{Al}$ & 0,0761 & 0,0000 & 0,0569 & 0,6712 & 0,0076 & 15,5352 & 15,6041 & 12,8329 & 12,9072 & 0,0075 & 0,0229 \\
\hline $\mathrm{Cr}$ & 0,0341 & 0,0000 & 0,0032 & 2,1801 & 0,0032 & 0,0077 & 0,0103 & 2,2701 & 2,1398 & 0,0065 & 0,0000 \\
\hline $\mathrm{Fe}^{3+}$ & 21,0408 & & & 17,3984 & & 0,5853 & 0,4137 & 1,1636 & 1,2344 & & \\
\hline $\mathrm{Fe}^{2+}$ & 10,5548 & & & 9,7486 & & 3,9222 & 3,8083 & 3,3531 & 3,4747 & & \\
\hline $\mathrm{Ni}$ & & & & & & 0,0150 & 0,0150 & & & & \\
\hline Mn & 0,0097 & 0,4345 & 0,3230 & 0,1281 & 0,1342 & 0,0324 & 0,0351 & 0,0425 & 0,0454 & 0,0065 & 0,2920 \\
\hline $\mathrm{Mg}$ & 0,0000 & 0,0000 & 0,0000 & 0,2326 & 0,3743 & 4,0914 & 4,1762 & 4,7345 & 4,6174 & 0,5401 & 0,1378 \\
\hline $\mathrm{Ca}$ & 0,0110 & 0,0108 & 0,7503 & 0,0155 & 0,0069 & 0,0031 & 0,0094 & 0,0032 & 0,0032 & 0,0069 & 0,0070 \\
\hline \multirow[t]{2}{*}{ Total } & 31,8346 & 22,0594 & 20,9274 & 30,4735 & 21,8095 & 24,2106 & 24,2106 & 24,4243 & 24,4495 & 21,6112 & 21,5988 \\
\hline & mag & ilm & ilm & mag-Cr & ilm & her & her & pic & pic & itm & ilm \\
\hline
\end{tabular}

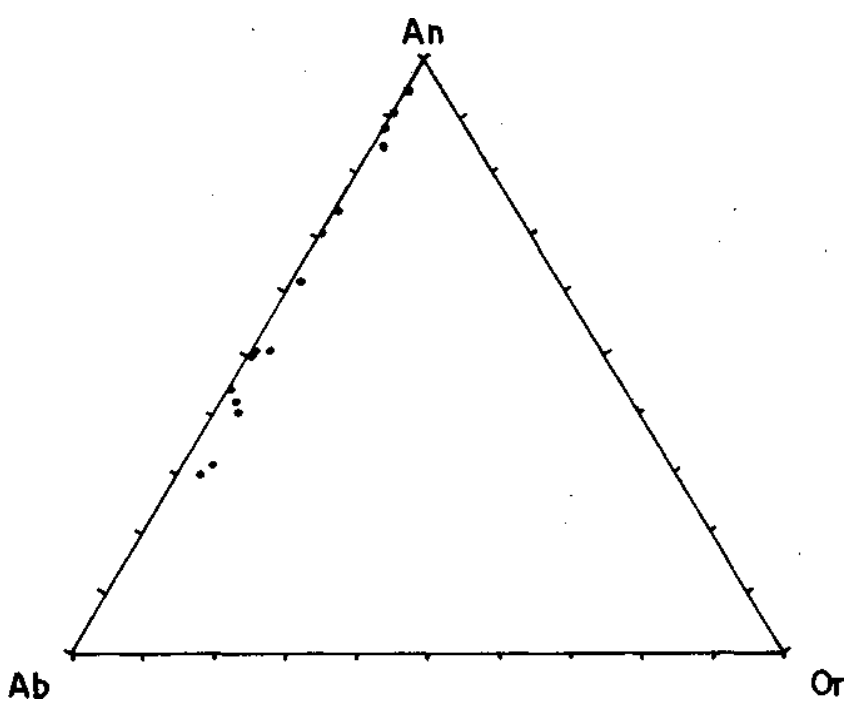

Figura 2 - Composição de plagioclásios

Figure 2 - Composition of plagioclase

cristalização dos piroxênios, em concordância com os cálculos barométricos.

A amostra CGSMC-33 contém a paragênese sillimanita, granada, plagioclásio, rutilo, quartzo e grafita. $\mathrm{O}$ plagioclásio e a sillimanita estão intensamente alterados para muscovita. O geobarômetro granada-plagioclásio (Newton \& Haselton 1981), em temperatura estipulada em $800^{\circ} \mathrm{C}$, forneceu pressão de 4,5 a 6,9 kbar. Estes valores não são concordantes com aqueles obtidos nos granulitos máficos, e seguem a tendência dos metassedimentos de fácies granulito de apresentarem resultados barométricos incorretos e baixos.

GEOTERMOMETRIA Os estudos termométricos realizados foram baseados nas paragêneses ortopiroxênio-clinopiroxênio, granada-clinopiroxênio e granada-hornblenda.

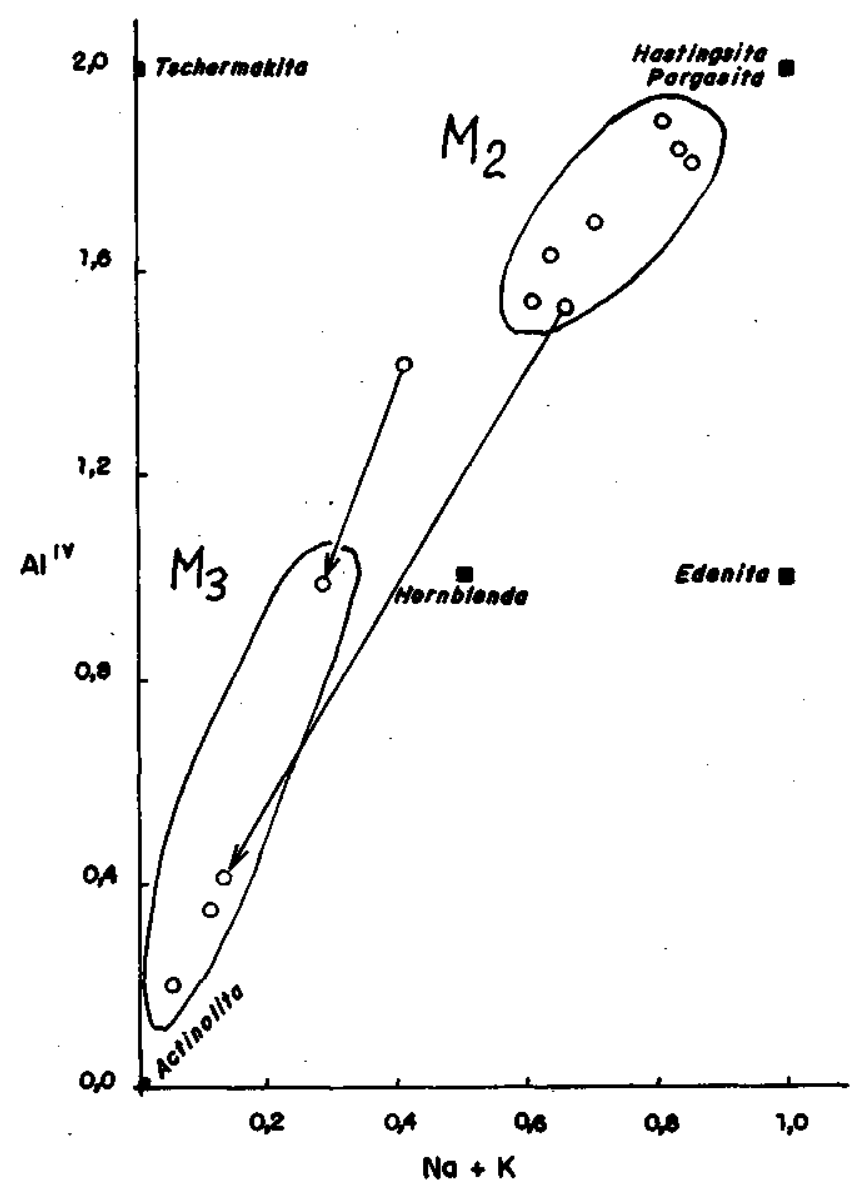

Figura 3 - Composição de aufibólios Figure 3 - Composition of amphiboles

Pares de piroxênios coexistentes foram analisados em várias amostras. Foram selecionados cristais em contato, per- 


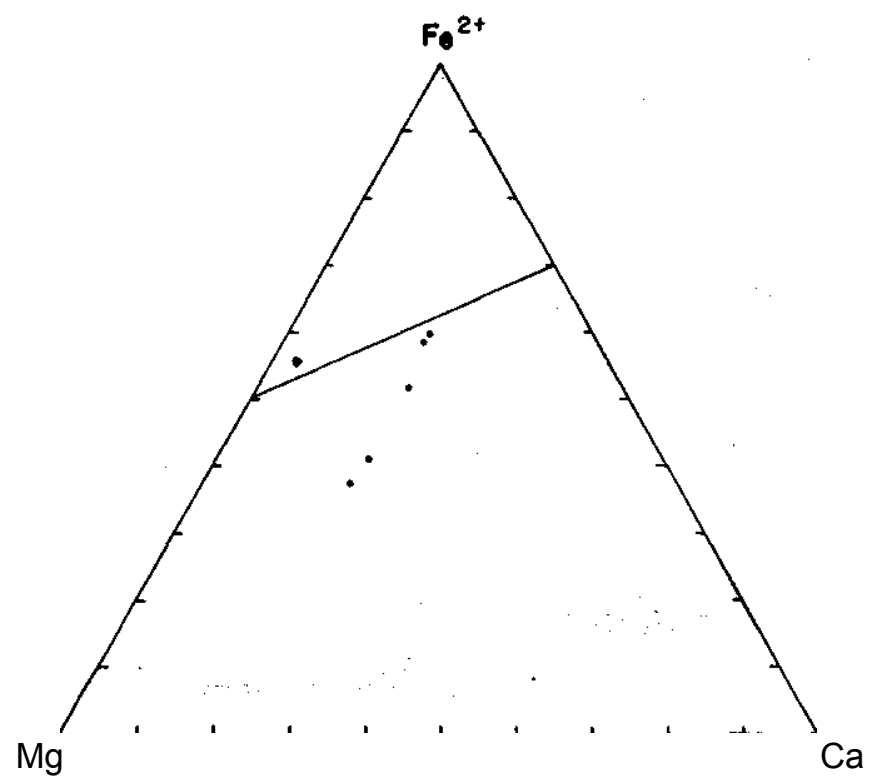

Figura 4 - Composição de granadas. A linlia reta representa o limite entre granadas mais ferrosas de terrenos de baixa pressão de outras regiões do mundo e granadas mais cálcio-magnesianas provenientes de terrenos granuliticos de altas pressões. As três amostras de granadas de granulitos básicos do Complexo Granulitico Santa Maria Chico caem no campo de alta pressão; uma amostra de granada de metapelito cai no outro campo

Figure 4 - Cornposition of garnets. Garnets from basic gtanulites from lowpressure granulite terroins from other parts of the world fali above the line (owards the iron-rich comer; lhose from high-pressure terrains fali below the line towards compositions more calcic-magnesian. The tliree samples of garnet from basic granulites from the Santa Maria Chico Granulite Complex fali in the high-pressure field; one sample of garnet from a metapelite falls ia the other field

tencendo a maioria ao evento $\mathrm{Ma}$; os cristais analisados na amostra CGSMC-37 são pequenos, podendo corresponder a um evento posterior a $\mathrm{M}_{2}$. Na tabela 7 , são apresentados os resultados dos cálculos termométricos, para um erro estimado de $5 \%$ no conteúdo de $\mathrm{Ca}$ do ortopiroxênio. Os termómetros a dois piroxênios indicam que o evento $\mathrm{M}_{2}$ ocorreu a cerca de $800^{\circ} \mathrm{C}$, mas o desvio padrão dos dados é muito elevado. A falta de consistência do termómetro torna sua validade restrita, podendo o resultado ser considerado apenas uma aproximação. A amostra CGSMC-37 indica uma temperatura da ordem de $700^{\circ} \mathrm{C}$

O par granada-clinopiroxênio ocorre na amostra CGSMC39 , fornecendo os resultados da tabela 8 . Este termômetro indica temperaturas da ordem de $800^{\circ} \mathrm{C}$ para o evento $\mathrm{M}_{2}$, principalmente quando se considera o termómetro desenvolvido por Ellis \& Green (1979).
Tabela 7 - Temperatura de recristalização do $M_{2}$, com base em dois piroxênios. A amostra CGSMC-37 corresponde a $M_{3}$ Table $7-\mathrm{M}_{2}$ recrystallization temperatures, based on two pyroxenes. An $\mathrm{M}_{3}$ crystal was analysed on sample CGSMC-37

\begin{tabular}{l|c|c|c|c|c|c|c|c|c}
\hline Aliostra CGSMC- & 1 & 17 & 25 & 31 & 35 & 36 & 37 & 39 & 40 \\
\hline Pressão eslipulada, kbar & 9,4 & 9,4 & 10 & 10 & 9,4 & 9,4 & 9,4 & 10 & 9,4 \\
Temperatura calculada, ${ }^{\circ} \mathrm{C}$ & & & & & & & & & \\
Wells (1977) & 829 & 782 & 835 & 940 & 822 & 764 & 696 & 871 & 845 \\
Wood \& Baino (1973) & 826 & 786 & 813 & 871 & 892 & 766 & 761 & 844 & 822 \\
\hline
\end{tabular}

Tabela 8 - Temperatura de $M_{2}$, calculada a partir de granada e clinopiroxênio na amostra CGSMC-39

Table $8-\mathrm{M}_{2}$ temperatures, calculated from garnet and clinopyroxene in sample CGSMC-39

\begin{tabular}{l|c|c|c}
\hline Pressão estipulada, kbar & 9,4 & 8 & 7 \\
Teimperatura calculada, ${ }^{\circ} \mathrm{C}$ & & & \\
Saxetis (1979) & 947 & 939 & 934 \\
Ellis \& Green (1979) & 815 & 810 & 806 \\
Dalıl (1980) & 863 & 852 & 846 \\
\hline
\end{tabular}

Graham \& Powell (1984) desenvolveram o termómetro granada-hornblenda pela comparação empírica com outros termómetros, e indicam sua utilização para rochas de alto grau. O par foi avaliado na amostra CGSMC-37, fornecendo a temperatura de $830^{\circ} \mathrm{C}$ para o evento $\mathrm{M}_{2}$. Este resultado está próximo ao obtido nos outros termómetros.

As temperaturas obtidas com o par magnetita-ilmenita foram muito baixas, da ordem de $500^{\circ} \mathrm{C}$, e devem corresponder ao reequilíbrio de baixo grau.

CONCLUSÕES A composição química dos minerais fornece informações significativas para a evolução do complexo. A composição cálcica do plagioclásio indica uma geração das litologias a partir de magmas básicos diferenciados, conforme já indicado pela geoquímica de rocha total.

Alguns dos resultados geotermobarométricos apresentam inconsistências, como a pressão obtida de granada-sillimanita-plagioclásio e a ampla variação de temperaturas obtida a partir de dois piroxênios. Mesmo assim, é possível concluir que o evento M2 atingiu aproximadamente condições de 9,4 kbar e $800^{\circ} \mathrm{C}$ no Complexo Granulítico Santa Maria Chico. $\mathrm{O}$ reajuste local do equilíbrio mineralógico parece ter ocorrido a cerca de $8 \mathrm{kbar}$ e $700^{\circ} \mathrm{C}$, possivelmente durante a ascensão epirogênica do complexo.

Agradecimentos O trabalho analíticoe cálculos foram realizados na Open University, Inglaterra, em 1985, encontrando-se o autor nessa oportunidade na condição de professor visitante na Universidade de Southampton. O apoio financeiro foi dado pelo Conselho Nacional do Desenvolvimento Científico e Tecnológico do qual o autor é pesquisador.

\section{REFERENCIAS BIBLIOGRÁFICAS}

CAWTHORN, R.G. \& COLLERSON, K.D. 1974. The recalculation of pyroxene end-member parameters and the estimation of ferrous and ferric iron content from electfon microprobe analyses. Am. Mineral., 59:1203-1208.

DAHL, P.S. 1980. The thermal-compositional dependence of Fe2+/Mg distributíons between coexisting garnets and piroxenes: applications to geothermometry. Am. Mineral., 65:852-866.

ELLIS, E.J. \& GREEN, D.H. 1979. An experimental study of the effect of Ca upon garnet-cíinopyroxene Fe-Mg exchange equilibria. Contrib. Mineral. Petrol, 71:13-22.

FIGUEIREDO, M.C.H. \& HARTMANN. L.A. 1989. Geoquímica dos elementos terras raras em granulitos e charnockitos do Brasil. In: FORMOSO, M.L.L.; NARDÏ, L.V.S. \& HARTMANN, L.A. Geoquímica dos Elementos Terras Raras no Brasil. SBGq/DNPM/CPRM. p. 99-105.
GRAHAM, C.M. \& POWELL, R 1984. A garnet-hornblende geothermometer: calibration, testing and application to the Pelona Schist, Southern Califórnia. .7. Metamorphic Geol, 2:13-31.

HARTMANN, L.A. 1987. Isócrona Sm-Nd de 2,1 Ga em minerais de duas amostras do Complexo Granulítico Santa Maria Chico, RS. In: CONGR. BRÁS. GEOQ., 1. Porto Alegre, 1987. Anais... Rio de Janeiro, SBGq. v. 1, p. 105-111.

HARTMANN, L.A. 1988. Geoquímica de terras raras e geotermobarometria de granulitos de Dom Pedrito e Luís Alves, no extremo sul do Brasil. Geoch. Bros. 2(1): 1-14.

JOST, H. \& HARTMANN, L.A. 1984. Província Mantiqueira - Setor Meridional. In: ALMEIDA, F.F.M. \& HASUI, Y. (coords.). $O$ Pré-Cambriano do Brasil. São Paulo, Edgard Blücher. p. 345-368.

NARDI. L.V.S \& HARTMANN, L.A. 1979. O Complexo Granulítico Santa Maria Chico do Escudo Sul-Riograndense. Acta Geol. Leop., 6:45-75. 
NAUMANN, M.P.; HARTMANN, L.A.; KOPPE, J.C.; CHEMALE. F. 1984. Sequências supra-crustais, gnaisses graníticos, granulitos e granitos intrusivos da região de Ibaré-Palma, RS: geologia, aspectos estratigráficos e considerações geotectônicas. In: CONGR. BRÁS. GEOL., 33. Rio de Janeiro, 1984. Anais... Rio de Janeiro, SBG. p. 2417-2425.

NEWTON, R.C. \& HASELTON, H.J. 1981. Thermodynamics of the garnet-plagioclase-AhSiOs quartz geobaromet.»-. In: NEWTONS, R.C. NAVROTSKY, A; WOOD, B.J. eds. Thermodynamics of Minerais and Melts. New York, Springer-Verlag. p. 125-145.

SANTOS, E.L.; SILVA, L.C.; ORLANDI, V.; COUTINHO, M.G.N.; R01SENBERG, A; RAMALHO, R. HARTMANN, L.A. 1984 Os Escudos Sul-Riograndense e Catarinense e a Bacia do Paraná. In: SCHOBBENHAUS, C.; CAMPOS, D.; DERZE, G.R.; ASMUS, H.E. (coofds.). Geologia do Brasil. DNPM.
SAXENA, S.K. 1979. Garnet-clinopyroxene geothermometer. Contrib. Mineral. Petroi, 70:229-235.

SOLIANI E Jr 1986 Os dados geocronológicos do Escudo Sul-RioGrandense e suas implicações de ordem geotectônica. São Paulo. 388 p. (Tese de Doutoramento, IG/USP).

WELLS, P.R.A. 1977. Pyroxene thermometry in simple and complex systems. Contrib. Mineral. Petroi., 62:129-139.

WOOD, B.J. \& BANNO, W. 1973. Garnet-orthopyroxene and orthopyroxene-clinopyroxene relationships in simple and complex systems. Contrib. Mineral. Petroi., 42:109-124.

MANUSCRITO A656 Recebido $\mathrm{cm} 27$ de abril de 1990 Revisão do autor em 05 de abril de 1991 Revisão do autor em 05 de abril de 1991
Revisão aceita em 27 de junho de 1991 